\title{
Editorial
}

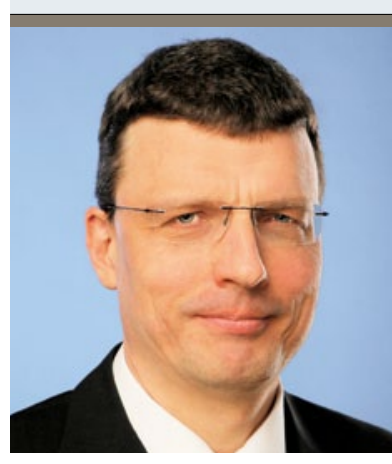

„Insgesamt kann die Auffassung vertreten werden, dass auch

Schlaganfallpatienten ohne Depression von einer pharmakologischen

Therapie mit SSRI profitieren."

Privatdozent Dr. med. Hans-Jörg Stürenburg,

Chefarzt für Neurologie, Bad Nenndorf

\section{Nach Schlaganfall profitieren alle Patienten von SSRI-Gabe}

$\mathrm{E}$ ines der Anliegen von DNP - Der Neurologe und Psychiater ist der Zusammenhalt zwischen Neurologie und Psychiatrie, obschon sich die Fächer immer weiter spezialisieren. Auch diese DNP-Ausgabe bündelt in hervorragender Art und Weise aktuelles Wissen um neurologische und psychiatrische Krankheitsbilder. Die Kombination beider „Flügel“ der Nervenheilkunde findet auch in die Forschung zur Rehabilitation nach Schlaganfall Eingang. Dabei rückt die gezielte neuropharmakologische Therapie immer stärker in unser Blickfeld. Denn dass serotonerge und noradrenerge Substanzen motorische Funktionsstörungen nach Schlaganfall verbessern können ist keineswegs nur mehr Theorie.

$\mathrm{Zu}$ den neueren Studien, die diese These untermauern, zählt beispielsweise die FLAME-Studie [1], die zeigte, dass Fluoxetin in der Frühphase nach Hirninfarkt die motorische Erholung bei mittelschweren bis schweren motorischen Defiziten deutlich verbessert. Zudem zeigte das Wissenschaftler-Team um Jorge et al., dass Escitalopram die globale kognitive Funktion und die verbalen und visuellen Gedächtnisleistungen nach Schlaganfall depressionsunabhängig verbessert [2]. Außerdem konnte in anderen Studien gezeigt werden, dass selektive SerotoninWiederaufnahmehemmer (SSRI) nicht nur in der Behandlung, sondern auch in der Prävention der Post-Stroke-Depression bei nicht-depressiven Patienten wirksam sind $[3,4]$.

\section{Kortikale Reorganisation und neuronale Plastizität}

Hinsichtlich der Post-Stroke-Depression räumt die Studie von Engelter et al. relevante Sicherheitsbedenken gegen eine pharmakologische Augmentation aus [5]. Die Autoren dieser Studie, in der innerhalb von 20 Monaten 97 (39\%) von 249 Schlaganfallpatienten mit pharmakologischer Augmentation behandelt wurden (32\% erhielten SSRI, die übrigen L-Dopa oder Acetylcholinesterase-Hemmer) empfehlen nach
Schlaganfall eine Augmentation mit SSRI. Denn Arzneien dieser Wirkstoffklasse beugen nicht nur einer (häufigen) Post-Stroke-Depression vor, sondern verbessern auch die kortikale Reorganisation und die neuronale Plastizität bei Patienten nach Schlaganfall, bei denen aktuell keine Depression vorliegt. Von kortikaler Reorganisation und neuronaler Plastizität profitieren auch Patienten mit Post-Stroke-Depression.

\section{Bestehende Medikation unbedingt überprüfen}

Auch wenn SSRI nach der oben genannten Studie ohne Sicherheitsbedenken augmentiert werden können, sollte nach Hirnschädigung prinzipiell immer auch die allgemeinmedizinische Medikation überprüft werden. Denn mögliche anticholinerge, antihistaminerge, anti-noradrenerge und dopaminantagonistische Effekte können die kortikale Reorganisation behindern. Besonders gefährlich erschienen Lazar et al. in diesem Zusammenhang die gabaergen Effekte etwa von Benzodiazepinen [6].

Insgesamt kann beim Thema „Rehabilitation nach Schlaganfall" die Auffassung vertreten werden, dass auch Schlaganfallpatienten ohne Depression von einer pharmakologischen Therapie mit SSRI profitieren, weil eine Verbesserung der motorischen Erholung und der kognitiven Funktion sowie die Prävention einer möglichen Post-Stroke-Depression bei akzeptabler Medikamentensicherheit zu erwarten sind. Nun ist es Aufgabe der Ärzteschaft, dieses Wissen auch in der Praxis umzusetzen.

\section{Praxisnahe Fortbildungen}

Neben aktuellen Berichten von der 51. Jahrestagung der Deutschen Gesellschaft für Epileptologie in Stuttgart und dem Europäischen Psychiatrie-Kongress in Prag finden Sie in dieser Ausgabe eine ganze Reihe erstklassiger Fortbildungsbeiträge. Von großer praktischer Relevanz ist zum Beispiel die CME-Fortbil- 


\section{Nach neueren Studien können SSRI wie Fluoxetin und Escitalopram nach Hirninfarkten sowohl die motorische Erholung bei mittel- schweren bis schweren motorischen Defiziten deutlich fördern als auch die verbalen und visuellen Gedächtnisleistungen. Außerdem können Sie der Entstehung einer Post-Stroke-Depression vorbeugen.}

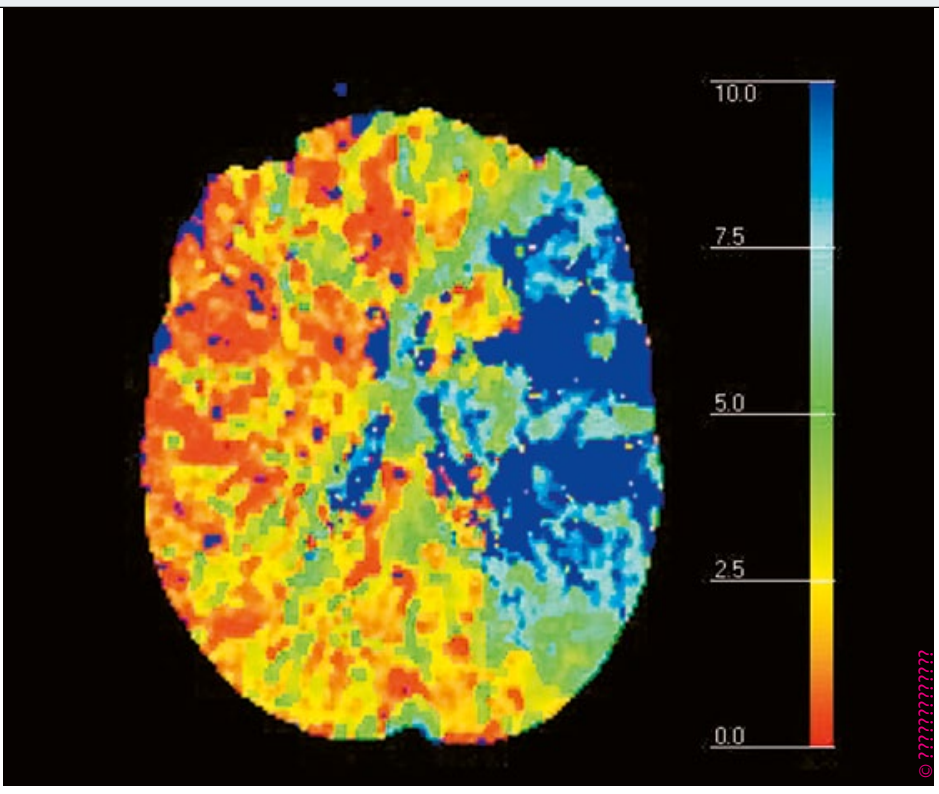

dung von Professor Haensch, Wuppertal, über die „Bedside“-Diagnostik zur Untersuchung des autonomen Nervensystems. Unter Betonung der speziellen Anamnese hinsichtlich autonomer Symptome zeigt der Artikel auf, dass ein effektives Screening auf vegetative Funktionsstörungen mit überschaubarem Aufwand möglich ist (siehe Seite 64).

Die CME-Fortbildung von Professor Brieger, Kempten, verdeutlicht, dass Psychoeduktion bei bipolar affektiven Störungen nicht nur sehr wirksam, sondern auch kostengünstig ist - und dies ohne Nebenwirkungen (siehe Seite 57).

Dass die Wirkung von Botulinumtoxin bei chronischer Migräne trotz unklaren Wirkmechanismus' als gesichert angenommen werden darf, legt Professor Jost, Wiesbaden, in einem prägnanten Fortbildungsbeitrag dar (siehe Seite 72). Darin wird aber auch deutlich, dass eine Differenzierung in „Responder" und „Non-Responder" klinischpraktisch sinnvoll ist.

Weiterhin finden Sie in dieser Ausgabe wertvolle Fortbildungen zum Thema „Multiple Sklerose“. Den aktuellen Stand der Dinge im dynamischen Feld der neuen MSTherapien haben Dres. Buttmann und Weise, Würzburg, im Beitrag „Welche Chancen bieten neue MS-Therapien“ (siehe Seite 76) für Sie zusammengefasst. Je mehr Therapeutika aus der Pipeline zur Zulassung gelangen, umso individueller wird die MS-Behandlung künftig gestaltet werden können. Darüber hinaus haben Professor Mäurer und Mitarbeiter einige wichtige neue Studien zur MS aus den zurückliegenden zwölf Monaten für Sie referiert und kritisch kommentiert (siehe Seite 26).

Die Plasmapherese wird nicht nur als eskalierende Schubtherapie bei der Multiplen Sklerose oder der Neuromyelitis optica eingesetzt, sondern auch in der Akuttherapie neuroimmunologischer Erkrankungen wie des Guillain-Barré-Strohl-Syndroms und der myasthenen
Krise. Privatdozent Dr. Linker, Erlangen, und Dr. Schröder, Bochum, erläutern das bei sachgerechter Anwendung sichere und gut verträgliche Verfahren (siehe Seite 90) und betonen, dass alle Neurologen und Nervenärzte mit den Indikationen zur therapeutischen Plasmapherese vertraut sein sollten, um geeignete Patienten dieser wirksamen Therapie zuzuführen.

Viel Spaß bei diesen praxisnahen, hervorragenden Fortbildungen wünscht Ihnen Ihr

Hans-Jörg Stürenburg

Priv.-Doz. Dr. med. Hans-Jörg Stürenburg

Chefarzt Neurologie

Klinik Niedersachsen

Hauptstr. 59, 31542 Bad Nenndorf

\section{Literatur}

1. Chollet et al. Fluoxetine for motor revovery after acute ischaemic stroke (FLAME): a randomised placebo-controlled trial. Lancet Neurol. 2011; 10 (2): 123-30

2. Jorge RE et al. Escitalopram and enhancement of cognitive recovery following stroke. Arch Gen Psychiatry. 2010; 67 (2): 187-96

3. Ramasubbu R. Therapy for prevention of post-stroke-depression. Expert Opin Pharmacother. 2011; 12 (14): 2177-87

4. Robinson RG et al. Escitalopram and problem-solving therapy for prevention of poststroke depression: A randomized controlled trial. JAMA 2008; 28: 299 (20): 2391-400

5. Engelter ST et al. Safety of pharmacological augmentation of stroke rehabilitation. Eur Neurol. 2010; 64 (6): 325-30

6. Lazar RM et al. GABAergic but not anti-cholinergic agents re-induce clinical deficits after stroke. J Neurol Sci. 2010; 15 : 292 (12): 72-6 